\title{
Chinese patent herbal medicine Huaiqihuang for Henoch-Schonlein purpura nephritis in children: a systematic review of randomized controlled trials
}

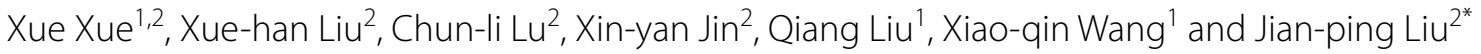

\begin{abstract}
Background: Henoch-Schönlein purpura nephritis (HSPN) is listed as the most common secondary glomerular diseases among children. Approximately 15 to $20 \%$ of children eventually could develop into chronic renal failure. Chinese patent herbal medicine Huaiqihuang $(\mathrm{HQH})$ has been widely used in children with HSPN. This study aimed to evaluate the effectiveness and safety of HQH for HSPN in children, so as to provide evidence for clinical use.

Methods: Randomized controlled trials (RCTs) on HQH for HSPN in children were searched in eight Chinese and English databases from their inception to December 2020. We included children with HSPN received HQH combined with conventional medicine. Cochrane "Risk of bias" tool was used to assess methodological quality, and "Grading of Recommendations Assessment, Development, and Evaluation (GRADE) approach" to summarize the certainty of evidence for main findings. Effect estimates were presented as risk ratio (RR), mean difference (MD) or standardized mean difference (SMD) with 95\% confidence interval (CI) in meta-analyses using RevMan 5.3. Data not suitable for statistical pooling were synthesized qualitatively.
\end{abstract}

Results: In total seven RCTs were identified. Compared with conventional medicine alone, $\mathrm{HQH}$ plus conventional medicine showed the better effect in improving clinical cure rate (RR $1.58 ; 95 \% \mathrm{Cl} 1.17$ to $2.14 ; n=6)$ and total effective rate (RR $1.34 ; 1.16$ to $1.54 ; n=6$ ); reducing urine sediment erythrocyte count (MD $-9.23 ;-10.76$ to $-7.69 ; n=3$ ) and urine $\beta 2$ micro-globulin level (MD $-0.09 ;-0.12$ to $-0.06 ; n=2)$. No serious adverse event was recorded in all included trials.

Conclusions: Limited evidence showed $\mathrm{HQH}$ combined with conventional medicine had a beneficial effect for children with HSPN, and the side effects were mild. HQH may be a promising complementary therapy. However, long term follow-up, high quality and multicenter RCTs are required to confirm the findings.

Keywords: Chinese patent herbal medicine, Huaiqihuang, Henoch-Schonlein purpura nephritis, Children, Randomized controlled trials, Systematic review

*Correspondence: Liujp@bucm.edu.cn

${ }^{2}$ Beijing University of Chinese Medicine, Centre for Evidence-Based

Chinese Medicine, Beijing 100029, China

Full list of author information is available at the end of the article

\section{Background}

Henoch-Schönlein purpura (HSP) is an immunoglobulin A mediated disease characterized by a generalized vasculitis mainly involving the skin, joints, gastrointestinal tract, and kidneys [1]. Among them, HSP nephritis (HSPN) is potentially the most severe complication [2], 
which usually manifests as gross hematuria, microscopic hematuria, microalbuminuria to massive proteinuria, and even renal failure [3]. A national cross-sectional survey in China reported that HSPN was listed as the most common secondary glomerular diseases among children [4]. Although most children with HSPN have a good chance of achieving a recovery, some may have a prolonged course of disease, approximately 15 to $20 \%$ of children will eventually develop chronic renal failure [5]. Thus, it is of great importance to pay enough attention to it.

There is no unified treatment plan for HSPN because of the diverse clinical manifestations and complex pathological classification of HSPN [5]. At present, the treatment mainly includes symptomatic treatment, angiotensin-converting enzyme inhibitors (ACEIs) or angiotensin receptor blockers (ARBs), glucocorticoid (GC) and immunosuppressant (IS) [6, 7]. However, the effectiveness is limited, and it is easy to relapse after discontinuation of medicine. Long-term use of GC and IS may cause serious side effects and toxicity. Consequently, it is necessary to seek alternative and supplementary therapies to treat HSPN in children in order to improve disease remission rate, reduce relapse and relieve side effects.

Huaiqihuang $(\mathrm{HQH})$ granule is a Chinese patent herbal medicine which contains Huaier, Gouqizi and Huangjing. All ingredients of $\mathrm{HQH}$ were shown in Table 1. All of these have been extensively used in China for thousands of years [8]. In recent years, $\mathrm{HQH}$ granules have been widely applied in the clinical treatment of various chronic kidney disease, including primary nephrotic syndrome, IgA nephropathy, HSPN and other primary and secondary kidney diseases [9-13]. Among them, a number of clinical trials have shown that $\mathrm{HQH}$ combined with conventional medicine has a significant effect on reducing proteinuria, mitigating hematuria, preventing relapse and relieving clinical symptoms in children with HSPN [13-19]. Combined with the results of animal studies and in vitro experiments, the clinical effectiveness of $\mathrm{HQH}$ may be related to a variety of mechanisms, including regulating immune response, reducing inflammatory damage, protecting podocytes, lightening the proliferation of glomerular mesangial cells and improving renal fibrosis [20-25]. Currently, only some small sample clinical trials have reported the effect of $\mathrm{HQH}$ on children with HSPN, and there is still a lack of systematic reviews on its overall effect and safety. As a result, this review aimed to review

Table 1 Ingredients of Huaiqihuang granule

\begin{tabular}{lll}
\hline Latin binomial name & Pinyin & Chinese name \\
\hline Trametes robiniophila Murr & Huaier & 槐耳 \\
Lycium barbarum & Gouqizi & 枸杞子 \\
Polygonati sibiricum & Huangjing & 黄精 \\
\hline
\end{tabular}

systematically and evaluate the effectiveness and safety of $\mathrm{HQH}$ on treating pediatric HSPN based on randomized controlled trials so as to provide statistically reliable evidence.

\section{Methods \\ Protocol and registration}

Methods of this review were specified in advance and documented in International Platform of Registered Systematic Review and Meta-analysis Protocols (INPLASY. $\mathrm{COM}$ ) on 31th of December 2020. (Registration number: 2020120148). The content of review was reported according to the Preferred Reporting Items for Systematic Reviews and Meta-Analyses (PRISMA-2009) [26].

\section{Eligibility criteria Types of studies}

Randomized controlled trials (RCTs) regardless of the blinding method were identified.

\section{Types of participants}

All children with HSPN under 14years old were included. No restriction on sex or race of participants. HSPN diagnosis was made according to Clinical Guidelinesa division of nephrology edited by Pediatrics Branch of Chinese Medical Association in 2009 [27]. That was, within 6 months of the course of HSP, hematuria and/ or proteinuria appeared, with or without hypertension, edema, and renal damage. "Hematuria" included gross hematuria or microscopic hematuria. Besides, "proteinuria" need meet any of the following: (1) urine routine protein was positive within 1 week for 3 times; (2) $24 \mathrm{~h}$ urine protein quantitative $>150 \mathrm{mg}$; (3) urine microalbumin was higher than the upper limit of normal value within 1 week for 3 times. The pathological diagnosis of HSPN referred to the classification of the International Study of Kidney Disease in Children (ISKDC), and was divided into I-VI grades [27].

\section{Types of intervention}

In addition to basic treatment (including: diet management, avoidance of allergens, anti-infection, anti-platelet aggregation, anti-allergy, ACEI / ARB, and other symptomatic treatments.), experimental group took " $\mathrm{HQH}$ " or "HQH combined with GC" or "HQH combined with GC and IS" treatments. While control group was administered "GC" or "GC combined with IS" on the basis of basic treatment (BT).

\section{Types of outcome measures}

Primary outcome measures: (1) clinical cure rate; (2) total effective rate. Secondary outcome measures: (1) $24 \mathrm{~h}$ urinary protein excretion; (2) urine sediment erythrocyte 
count; (3) urine $\beta 2$ micro-globulin( $\beta 2-\mathrm{MG}$ ); (4) immune cells and inflammatory factors; (5) adverse events.

"Clinical cure" referred to the disappearance of symptoms and signs(purpura of the skin, joint swelling and pain, melena, hematuria, edema, hypertension.); urinary protein and urinary sediment erythrocyte continued to turn negative; or urinary protein quantitative $<0.15 \mathrm{~g} / 24 \mathrm{~h}$ [28]. "Clinical remission" referred to significant improvement in clinical symptoms and signs; quantitative reduction of urine protein $\geq 50 \%$; reduction of urine sediment erythrocyte count $\geq 50 \%$, or reduction of urine protein and urine occult blood $\geq$ " $2+$ " $[18,28]$. "Clinical cure rate" was defined as the number of clinical cures divided by the total number of participants. As well as definition of "partial remission rate" was the number of clinical remissions divided by the total number of participants. The sum of "clinical cure rate" and "partial remission rate" were "total effective rate".

\section{Exclusion criteria}

(1) Participants included in RCTs had serious complications or other serious diseases (autoimmune disease, connective tissue disease, hemopathy, tumor, liver disease, heart failure, myocardial infarction, infectious disease, organ transplants and so on). (2) Participants included in RCTs received hemodialysis or peritoneal dialysis treatment. (3) Any Chinese herbal medicine preparations (oral and topical) other than $\mathrm{HQH}$ in the study, including single herbs, compound herbal medicines, herbal extracts, raw materials, Chinese patent herbal medicines and herbal formulas prescribed by practitioners. (4) Unable to acquire full text of the publications.

\section{Search strategy}

We searched the following eight Chinese and English databases from their inception to December 2020. Four Chinese databases included China National Knowledge Infrastructure (CNKI), Wan Fang, Chinese Science and Technology Journal Database (VIP), and SinoMed Database. Four English databases included PubMed, EMBASE, the Cochrane Library, and Web of Science. Two trial registers including Clinical Trials. gov and the World Health Organization International Clinical Trials Registry Platform were also searched. Additionally, related reviews, conference papers, references lists and gray literatures also were searched manually to minimize the missed detection rate. No language or publication type was imposed. Taking "PubMed" as an example, the retrieval strategy was as follows: \#1: 'Purpura, Schoenlein-Henoch'[Mesh], \#2: 'Nephritis'[Mesh], \#3: \#1 AND \#2, \#4: 'Henoeh-Sehonlein purpura nephritis', \#5: 'purpura nephritis', \#6: 'HSPN',\#7: 'Immunoglobulin A vasculitis with nephritis', \#8: 'IgAVN', \#9: \#3 OR \#4 OR \#5 OR \#6 OR \#7 OR \#8, \#10: 'child'[Mesh], \#11: 'children', \#12: \#10 OR \#11,\#13: \#9 AND \#12, \#14: 'Huaiqihuang', \#15: 'HQH', \#16: \#14 OR \#15, \#17: \#13 AND \#16.

\section{Studies selection and data extraction}

The titles and the abstracts were screened first, then the full-text versions were checked according to inclusion and exclusion criteria. Two authors examined the full text to identify the eligible trials independently. We made a data extraction sheet in advance. Two evaluators extracted data independently. Disagreements of studies selection and data extraction were resolved by the corresponding author JP Liu. Information was extracted from each included trial on: (1) basic characteristics of the literature, including the first author, publication year, sample size of each group, intervention (including types of intervention; names, doses, duration and frequency of $\mathrm{HQH}$ ). (2) methodological characteristics of the trials. (3) types of primary and secondary outcome measure, length of follow up and adverse events reported.

\section{Quality assessment \\ Risk of bias in included RCTs}

Two authors assessed and validated the quality of included trials independently according to Cochrane Handbook for Systematic Reviews of Interventions [29]. Seven items including random sequence generation, allocation concealment, blinding of participants and personnel, blinding of outcome assessment, incomplete outcome data, selective reporting and other bias (pharmaceutical funding and comparability of baseline data of subjects between groups), were used to be judged as "unclear risk", "low risk", or "high risk". Any disagreements were resolved by discussion with the corresponding author JP Liu.

\section{Certainty of evidence for main findings}

Two reviewers summarized the certainty of evidence for main findings using the "Grading of Recommendations Assessment, Development, and Evaluation (GRADE) approach", independently. The certainty of evidence for the main findings was graded as GRADE working Group grades of evidence. "High certainty": very confident that the true effect size was close to the effect estimate. "Moderate certainty": have a moderate degree of confidence in the estimated value of effect; the true value may be close to the estimated, but there was still the possibility that the two were significantly different. "Low certainty": limited confidence in the estimated value of the effect; the true value may be very different from the estimated. "Very low certainty": there was little confidence in the 
effect estimate, and the true effect was likely to be very different from the estimated [30].

\section{Statistical analysis}

RevMan 5.3 software was used for data analysis. For continuous data, we used mean difference (MD) with 95\% confidence intervals (CI) or standardized mean difference (SMD) with 95\% CI (When the measurement unit of outcome was different, in order to eliminate the influence of the dimension, SMD can be selected). And for dichotomous data, we used risk ratio (RR) with $95 \%$ CI. We performed meta-analyses for trials if the study design, interventions, control and outcome measures were similar. Other data not suitable for pooling analysis were synthesized qualitatively.

I-square $\left(\mathrm{I}^{2}\right)$ was used to test the statistical heterogeneity as recommended by the Cochrane Handbook for Systematic Reviews of Interventions [31]. A rough interpretation of $\mathrm{I}^{2}$ statistic value in the context of meta-analyses of randomized trials was as follows: 0 to 40\%: might not be important; 30 to $60 \%$ : may represent moderate heterogeneity; 50 to $90 \%$ : may represent substantial heterogeneity; 75 to $100 \%$ : considerable heterogeneity. To reduce clinical heterogeneity, we conducted subgroups analyses of different interventions. We used random effects model for data pooling with moderate statistical heterogeneity (if $\mathrm{I}^{2}>30 \%$ ), otherwise a fixed effect model was applied if the trials were basically homogeneous (if $\mathrm{I}^{2} \leq 30 \%$ ). In case of the data were available, we did sensitivity analysis to explore the robustness of results. And we did funnel plots to test for the possibility of publication bias if there were enough studies (generally, more than 10 trials).

\section{Results \\ Search results}

One hundred and twenty-five relevant citations were retrieved from the searches. After scanning the full texts, two papers were excluded. In Wang N's article [32], the 'randomly allocate' was grouped according to the order of visits. Single numbers were the $\mathrm{HQH}$ group, and double numbers were conventional treatment group. And in study of Ma XL [33], the grouping method was unknown. Finally, seven papers were eligible for this review. All of them were published in Chinese. Details of search and selection process are illustrated in Fig. 1.

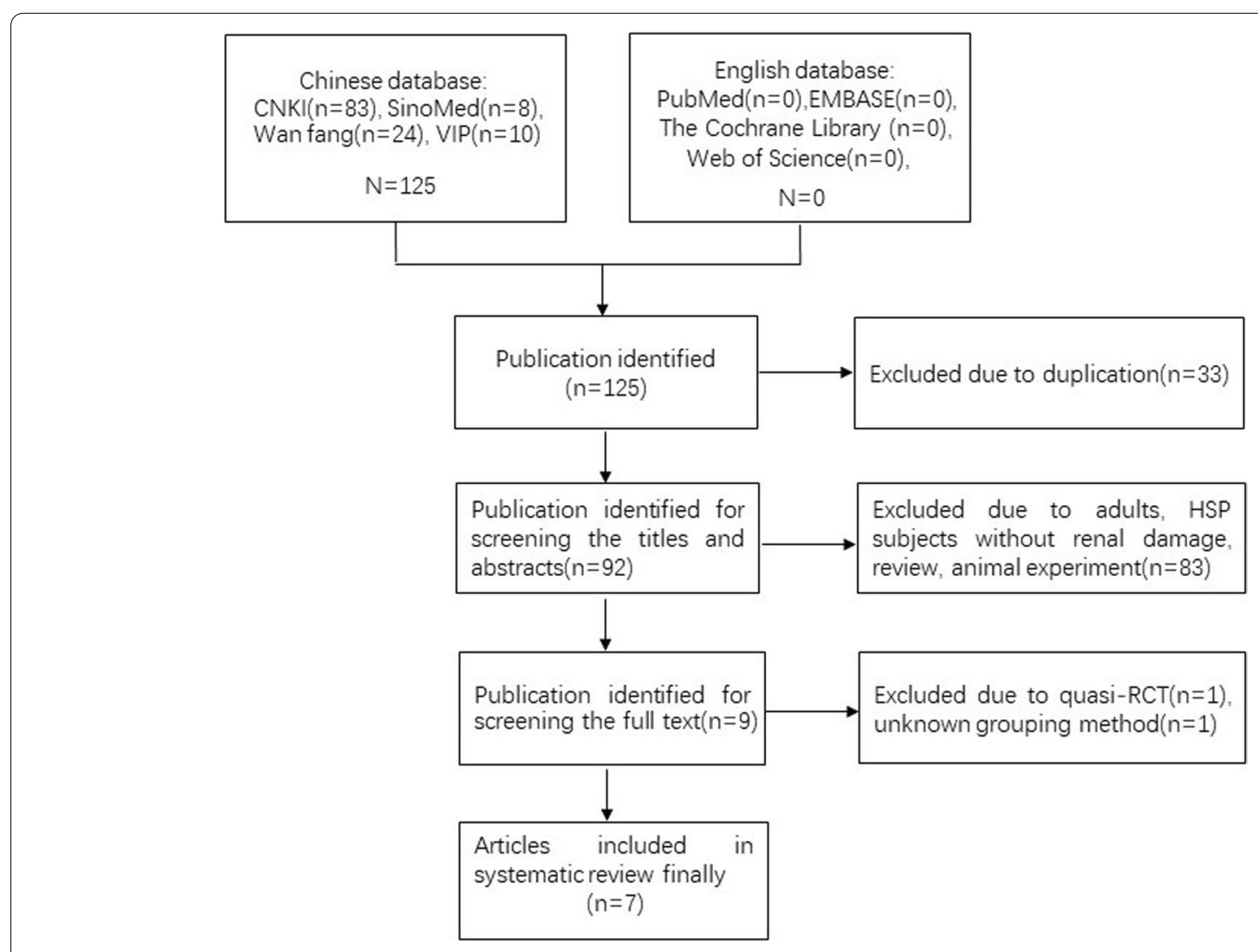

Fig. 1 Flow diagram of study search and selection 


\section{Characteristic of included trials}

A total of seven RCTs involving 393 participants were included [13-19]. There was no incidence of end-stage renal disease, hemodialysis, peritoneal dialysis or mortality reported among all the trails. Among them, Zhou DJ's citation was a stratified randomized controlled trial [19]. According to the random number table, 20 children with HSPN who were pathologically diagnosed as grade II were randomly divided into "basic treatment (BT)" group and "BT plus HQH" group, with 10 cases in each group. Also according to the random number table, 10 children with grade IIIa pathological examination were randomly divided into "GC" group and "GC plus $\mathrm{HQH}$ " treatment group, with 5 cases in each group. We used "Zhou DJ a" and "Zhou DJ b" to represent respectively. The main characteristics of included trials were demonstrated in Table 2.

\section{Risk of bias of included trials}

All trials indicated "random allocation". Three trials used the random number table [17-19], the remaining RCTs did not explain specific random methods [1316]. The trials only describing "randomized allocation" were defined as "unclear risk" of bias, while the trials reporting a specific method of randomized sequences generation as "low risk". No study provided sufficient information about allocation concealment. And "allocation concealment" of all trials were defined as "unclear risk" of bias. In addition, regarding blinding methods, no study used placebo granules. When the outcome measures of the study were only objective laboratory indicators, we defined performance bias and detection bias as "low risk", as well as when the outcomes of the study included subjective indicators, we defined as "high risk". No trial reported the number of participants lost to follow-up, so attrition bias was considered to be "low risk". As the research protocol of each study was not obtained, we regarded reporting bias as "unclear risk" at last. All included studies did not receive support from pharmaceutical funding and no statistical difference was found in baseline characteristics between groups, so we considered other biases to be "low risk". Despite we tried our best to contact authors by email or phone, no more information was obtained. In short, the included trials were evaluated for low methodological quality using a risk of bias assessment tool (Fig. 2).

\section{Outcome data and effects of interventions Primary outcome measures}

Clinical cure rate Six trials [13-18] reported clinical cure rate. Among them, 184 cases in experimental group, and 179 cases in control group. As presented in Fig. 3, the forest plot showed that compared with children treated with "conventional medicine" alone, children treated with "HQH combined with conventional medicine" had a more significant increase in clinical cure rates (RR 1.58; 95\%CI 1.17 to 2.14 ). The statistical heterogeneity of each study was moderate $\left(\mathrm{I}^{2}=33 \%\right)$. And taking into account the clinical heterogeneity of different interventions, we used random effect model. Additionally, we divided into two subgroups according to different conventional medicine interventions. Meta-analysis results of subgroups demonstrated that clinical cure rate of "GC plus $\mathrm{HQH}$ " group was better than "GC" group (RR $1.41 ; 1.06$ to 1.89 ). It was not found that clinical cure rate of "GC plus IS plus HQH" group was higher than that of "GC plus IS" group (RR 2.02; 0.97 to 4.18 ).

Total effective rate Six trials [13-18] reported total effective rate. Among them, 184 cases in experimental group, and 179 cases in control group. As shown in Fig. 4, the forest plot pointed that compared with children treated with "conventional medicine" alone, children treated with "HQH combined with conventional medicine" had a more obvious improvement in total effective rates ( $R R$ 1.34; 1.16 to 1.54 ). The statistical heterogeneity of each study was moderate $\left(\mathrm{I}^{2}=33 \%\right)$. And taking into account clinical heterogeneity of different interventions, we applied random effect model. In addition, we divided into two subgroups according to different conventional medicine interventions. Meta-analysis results of subgroups indicated that total effective rate of "GC plus HQH" group was better than "GC" group (RR $1.29 ; 1.10$ to 1.50$)$. And children treated with "GC plus IS plus HQH" also suggested a more obvious elevation in total effective rates when compared with "GC plus IS" (RR $1.52 ; 1.02$ to 2.26 ).

\section{Secondary outcome measures}

Urinary protein level Four trials [14, 17-19] compared the effectiveness on reducing urinary protein between experimental and control group. Among them, studies of Shi Z [17] and Zhou DJ [19] reported the urinary albumin excretion $(\mathrm{mg} / \mathrm{L}$ and $\mathrm{mg} / \mathrm{kg})$, all the others $[14$, 18] reported $24 \mathrm{~h}$ urinary protein $(\mathrm{g} / 24 \mathrm{~h})$. Due to different measurement units and considerable heterogeneity after pooling all results $\left(\mathrm{I}^{2}=90 \%\right)$, we abandoned the overall pooling. We reported the result of each trial separately. In the trials of Han DX [14], Shi Z [17] and Yuan TT [18], additional administration of $\mathrm{HQH}$ had a superior effectiveness compared to "GC plus IS" on reducing 


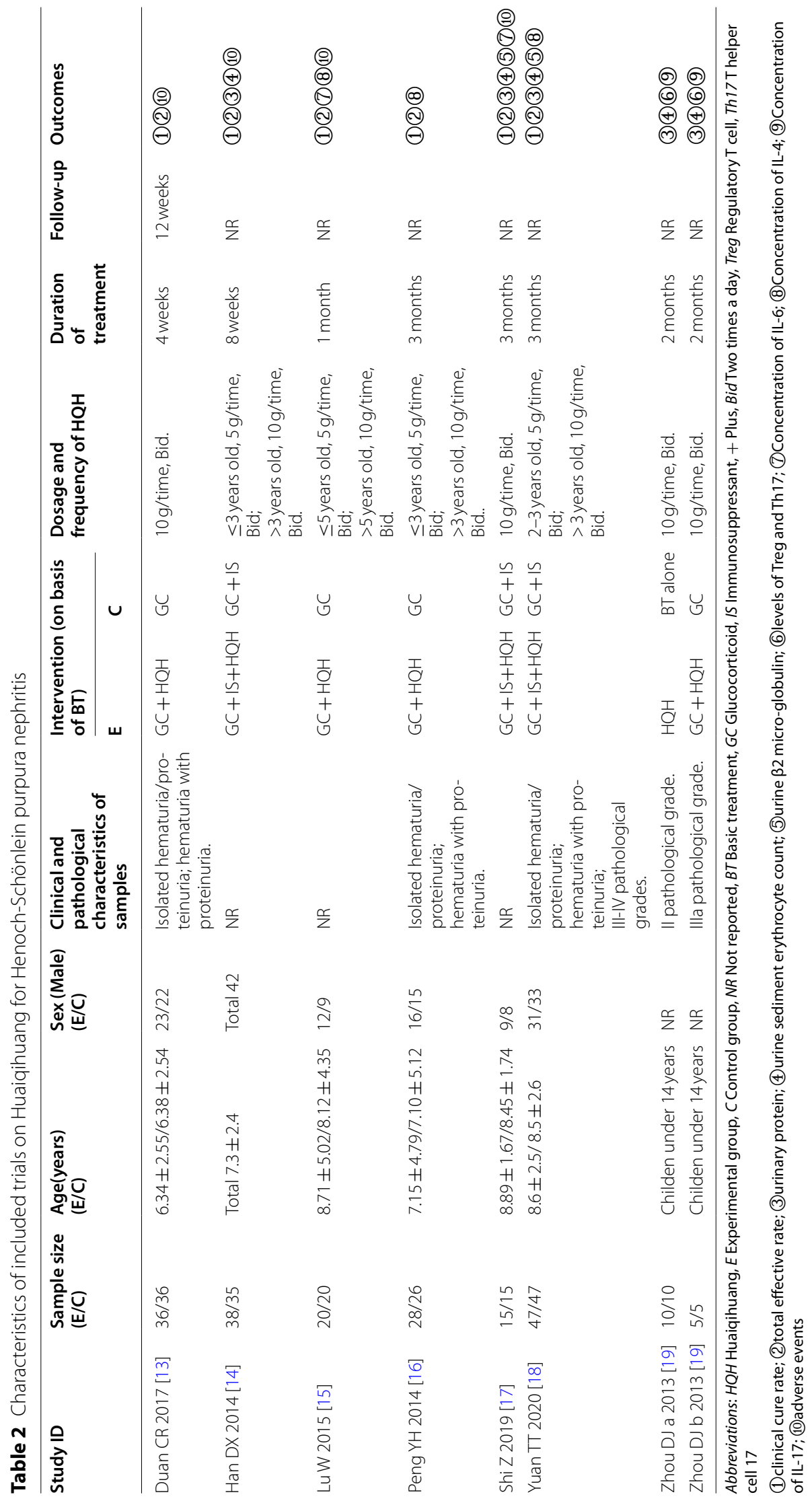




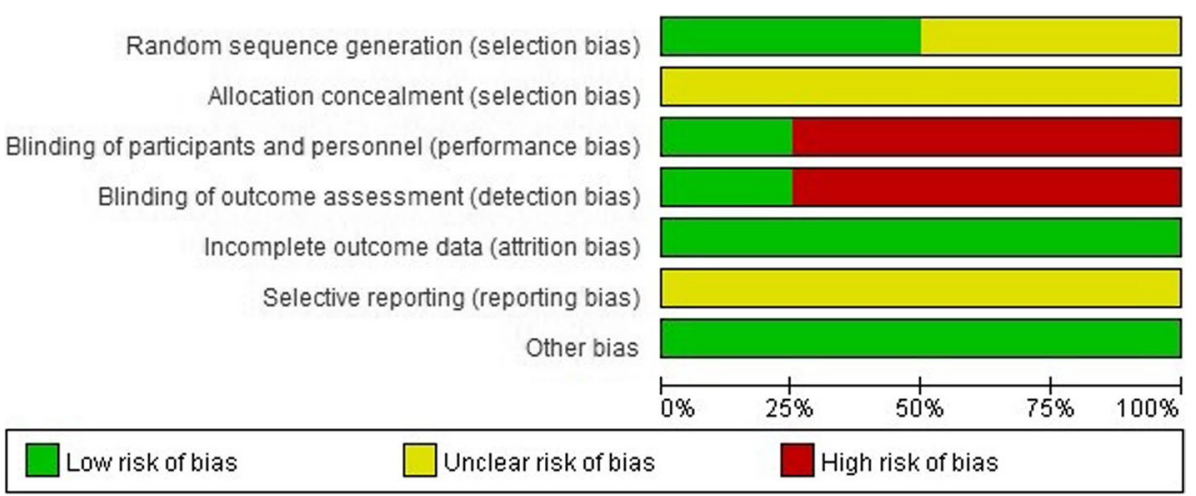

Fig. 2 Risk of bias graph: review authors'judgements about each risk of bias item presented as percentages across all included trials

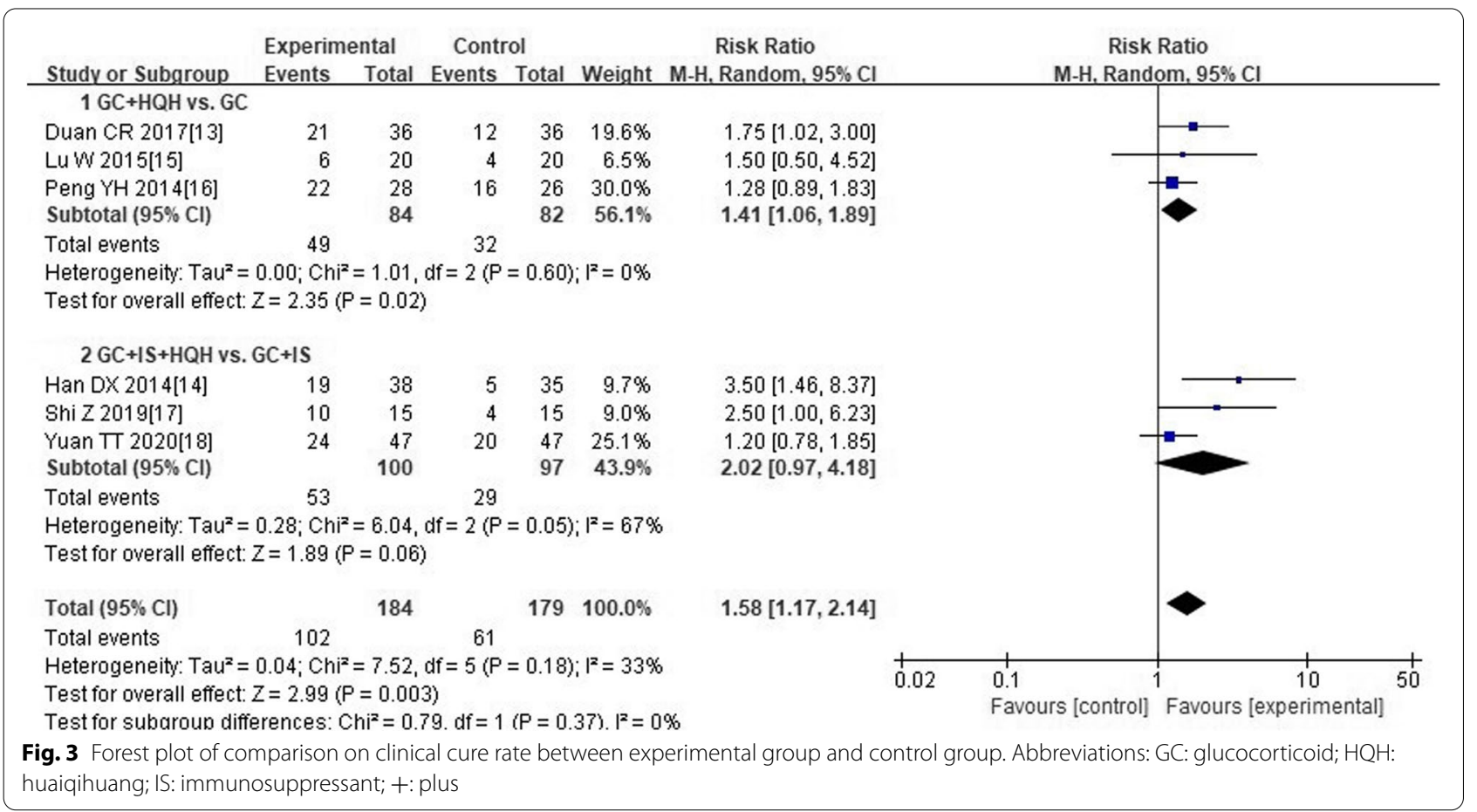

proteinuria, with a MD (and 95\%CI) of $-3.52(-4.26$ to -2.77$)$; $-1.70(-2.55$ to -0.85$)$ and $-0.92(-1.35$ to $-0.49)$, respectively.

In Zhou DJ's trial [19], there was no significant statistical difference between "HQH" group and "BT" group in lowering proteinuria, with a $\mathrm{MD}$ of -1.28 , and $95 \% \mathrm{CI}$ (-2.83 to 0.27$)$. While, "HQH plus GC" had a better effectiveness compared to "GC" group, with a MD of -6.05 , and $95 \%$ CI $(-9.86$ to -2.24$)$.

Urine sediment erythrocyte count Four trials [14, 17-19] compared the effectiveness on reducing urine sediment erythrocyte count between the experimental and control group. Among them, trial of Zhou DJ [19] reported the urine sediment erythrocyte by unit of " $\times 10^{7} \mathrm{Pcs} / \mathrm{L}$ ", all the others $[14,17,18]$ described by "Pcs/HP". As a result of considerable heterogeneity after pooling all results $\left(\mathrm{I}^{2}=95 \%\right)$, so we quitted the total pooling. We divided the different subgroups in accordance with different conventional medicine interventions. As shown in Fig. 5, the pooled result of 3 RCTs $[14,17$, 18] manifested that additional administration of $\mathrm{HQH}$ had a superior effectiveness compared to "GC plus IS" on reducing urine sediment erythrocyte (MD -9.23; -10.76 to -7.69 ). 


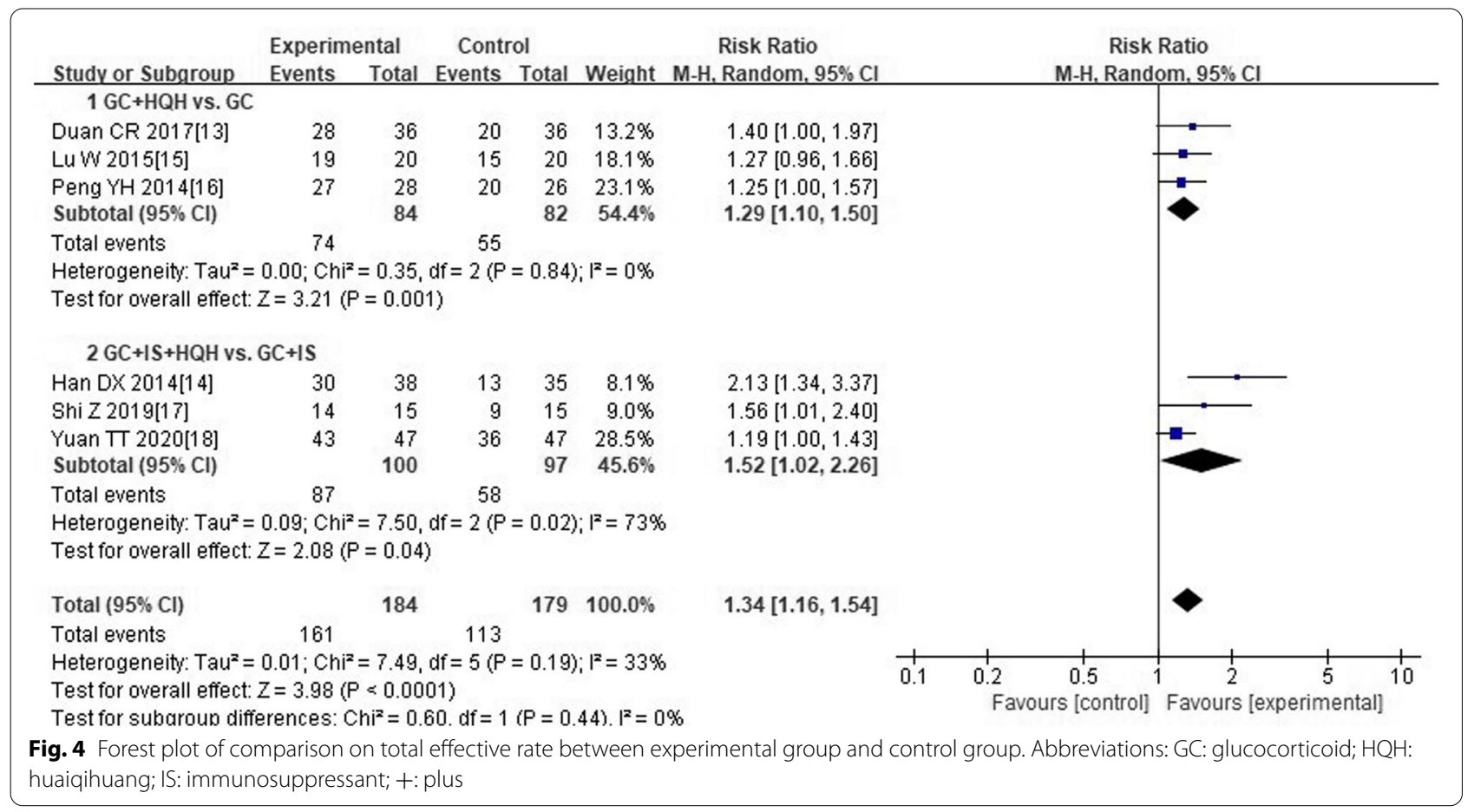

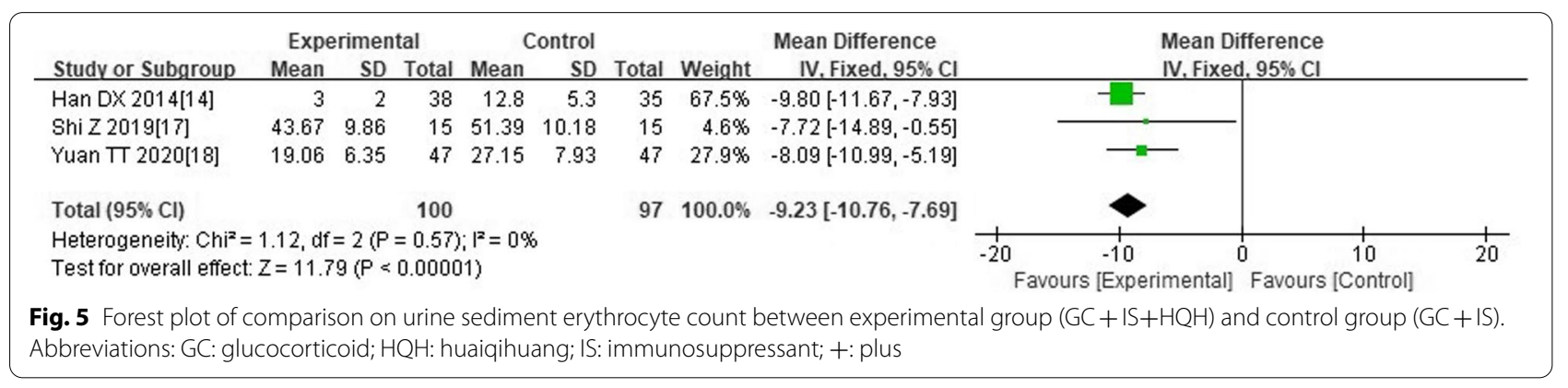

In Zhou DJ's trial [19], there was no obvious statistical difference between "HQH" group and "BT" group in lowering urine sediment erythrocyte, with a MD of 0.31 , and $95 \%$ CI ( -1.24 to 1.86$)$. In addition, the results suggested "GC plus HQH" group had a superior effectiveness compared to "GC" group, with a MD of -6.00 , and $95 \% \mathrm{CI}$ $(-9.22$ to -2.78$)$.

Urine $\beta 2$ micro-globulin( $\beta 2-M G)$ Two trials $[17,18]$ published urine $\beta 2-\mathrm{MG}$. There were 62 subjects in experimental group and 62 cases in control group. The unit of measurement for urine $32-\mathrm{MG}$ in both two trials was "mg/L". The pooled analysis indicated that "GC plus IS plus HQH" group had a better effect compared to "GC plus IS" group in decreasing urine $\beta 2-\mathrm{MG}$ level (MD
$-0.09,-0.12$ to -0.06$)$. Heterogeneity of studies might be not important $\left(\mathrm{I}^{2}=0 \%\right)$, and fixed effect model was selected.

Immune cells and inflammatory factors Levels of regulatory $T$ cell (Treg) and $T$ helper cell 17(Th17) Zhou DJ's trial [19] published outcomes of immune cells. The levels of Treg and Th17 were reported. The pooled result showed that compared with conventional medicine alone, co-intervention of $\mathrm{HQH}$ and conventional medicine had larger effect on elevating Treg (\%) level (MD $5.02 ; 4.15$ to 5.90$)$. The statistical heterogeneity of each study might not be important $\left(\mathrm{I}^{2}=0 \%\right)$. Considering the 
clinical heterogeneity, results of subgroups analyses were shown in Table 3.

Besides, the pooled result displayed that the function of lowering Th17(\%) levels were more apparent after additional administration of $\mathrm{HQH}$ than conventional medicine alone (MD - $0.75 ;-1.07$ to -0.43 ). The heterogeneity of each study represented substantial heterogeneity $\left(\mathrm{I}^{2}=56 \%\right)$. Thus, we performed subgroups analysis, and results were presented in Table 3.

\section{Concentration of Interleukin-6/Interleukin-4/Inter-} leukin-17 Two trials $[15,17]$ reported concentration of interleukin 6 (IL-6), both of which were measured in "pg/ $\mathrm{mL}$. The pooled result suggested that additional administration of HQH had a better effectiveness on decreasing IL-6 levels compared with "conventional medicine" alone (MD $-4.30 ;-6.70$ to -1.91$)$. The heterogeneity of each study might not be important $\left(\mathrm{I}^{2}=0 \%\right)$, and fixed effect model was adopted. A total of three trials [15, $16,18]$ published concentration of interleukin 4 (IL-4). Among them, researches of Lu W [15] and Peng YH [16] both used "pg/mL" as the unit of measurement, while Yuan TT's trial [18] adopted " $\mu \mathrm{g} / \mathrm{L}$ " to measure IL-4. The pooled result of three trials showed that the effect of reducing IL-4 was more significant after adding $\mathrm{HQH}$ than conventional medicine alone (SMD $-0.85 ;-1.15$ to $-0.54)$. The heterogeneity of each study might not be important $\left(\mathrm{I}^{2}=4 \%\right)$, and fixed effect model was used. The concentration of IL-17 was described in citation of Zhou DJ [19], which was measured in "ng/L". The pooled result of Zhou DJa and Zhou DJb indicated that the effect of reducing IL-17 was more significant after adding HQH than conventional medicine alone (MD -10.52; -19.12 to $-1.92)$, with a moderate heterogeneity $\left(\mathrm{I}^{2}=41 \%\right)$. Taking clinical heterogeneity into account, results of subgroups analyses were illustrated in Table 3.

Adverse events Only four trials $[13-15,17]$ reported the outcome of adverse events. Among them, one trial [13] described that there was no adverse event both in experimental group and control group throughout the whole study. Adverse events reported in the remaining three studies $[14,15,17]$ were shown in Table 4 . We did not acquire haematological biochemical record (blood routine, liver function) and any details about the treatment for adverse events from all included studies. None of the trials reported occurrence of serious adverse events such as permanent disability or death. As revealed in Fig. 6, the pooled analyses indicated that there was no significant statistical difference between "experimental group (co-intervention of $\mathrm{HQH}$ and conventional medicine)" and "control group (conventional medicine alone)" in incidence of adverse events, with an RR of 0.43 and a $95 \% \mathrm{CI}$ (0.15 to 1.24$)$.

\section{Certainty of evidence for main findings}

Combined with GRADE assessment results, the overall certainty of evidence for main findings were not good. Ranged from "very low" to "moderate". In most cases, certainty of evidence was downgraded owing to serious limitations of primary studies on design and execution. Furthermore, as a result of fewer than 10 articles were included, no publication bias test was performed, we downgraded it by one level finally (Table 5 ).

As shown in Table 5, only few studies with small sample size included in the key clinical questions. Considering that small sample size of the included studies

Table 3 Subgroups analyses of different interventions on "immune cells and inflammatory factors" outcomes

\begin{tabular}{|c|c|c|c|c|c|c|c|c|}
\hline \multirow[t]{2}{*}{ Outcomes } & \multicolumn{2}{|c|}{$\begin{array}{l}\text { Intervention (on basis } \\
\text { of BT) }\end{array}$} & \multirow[t]{2}{*}{$\begin{array}{l}\text { Sample size } \\
(E / C)\end{array}$} & \multirow[t]{2}{*}{ No. of studies } & \multirow[t]{2}{*}{$1^{2}$} & \multirow[t]{2}{*}{ Effect model } & \multirow[t]{2}{*}{$\begin{array}{l}\text { Effect estimation } \\
\text { MD; }(95 \% \mathrm{Cl})\end{array}$} & \multirow[t]{2}{*}{$P$ value } \\
\hline & $\mathrm{E}$ & $\mathrm{C}$ & & & & & & \\
\hline \multirow[t]{2}{*}{ Treg (\%) [19] } & $\mathrm{HQH}$ & BT alone & $10 / 10$ & $1[19]$ & - & - & $5.04 ;(4.15,5.93)$ & $P<0.00001$ \\
\hline & $\mathrm{GC}+\mathrm{HQH}$ & GC & $5 / 5$ & $1[19]$ & - & - & $4.42 ;(-1.20,10.04)$ & $P=0.12$ \\
\hline \multirow[t]{2}{*}{ Th17 (\%) [19] } & $\mathrm{HQH}$ & BT alone & $10 / 10$ & $1[19]$ & - & - & $-0.89 ;(-1.25,-0.53)$ & $P<0.00001$ \\
\hline & $\mathrm{GC}+\mathrm{HQH}$ & GC & $5 / 5$ & $1[19]$ & - & - & $-0.32 ;(-0.96,0.32)$ & $P=0.33$ \\
\hline \multirow[t]{2}{*}{ IL-6 $[15,17]$} & $\mathrm{GC}+\mathrm{HQH}$ & GC & $20 / 20$ & $1[15]$ & - & - & $-3.85 ;(-6.67,-1.03)$ & $P=0.007$ \\
\hline & $\mathrm{GC}+\mathrm{IS}+\mathrm{HQH}$ & $\mathrm{GC}+\mathrm{IS}$ & $15 / 15$ & $1[17]$ & - & - & $-5.49 ;(-10.04,-0.94)$ & $P=0.02$ \\
\hline \multirow[t]{2}{*}{ IL-4 $[15,16,18]$} & $\mathrm{GC}+\mathrm{HQH}$ & GC & $48 / 46$ & $2[15,16]$ & $61 \%$ & Random & $-4.91 ;(-8.80,-1.01)$ & $P=0.01$ \\
\hline & $\mathrm{GC}+\mathrm{IS}+\mathrm{HQH}$ & $\mathrm{GC}+\mathrm{IS}$ & $47 / 47$ & $1[18]$ & - & - & $-0.08 ;(-0.11,-0.05)$ & $P<0.00001$ \\
\hline \multirow[t]{2}{*}{ IL-17 [19] } & $\mathrm{HQH}$ & BT alone & $10 / 10$ & $1[19]$ & - & - & $-14.06 ;(-21.74,-6.38)$ & $P=0.0003$ \\
\hline & $\mathrm{GC}+\mathrm{HQH}$ & GC & $5 / 5$ & $1[19]$ & - & - & $-5.08 ;(-16.24,6.08)$ & $P=0.37$ \\
\hline
\end{tabular}

Abbreviations: Treg Regulatory T cell, Th17T helper cell 17, IL Interleukin, E Experimental group, C Control group, BT Basic treatment, GC Glucocorticoid, HQH Huaiqihuang, IS Immunosuppressant, + Plus, $I^{2}$ I-square, MD Mean difference, Cl Confidence interval 
Table 4 Adverse events of included trials on Huaiqihuang for Henoch-Schönlein purpura nephritis

\begin{tabular}{|c|c|c|c|c|}
\hline Study ID & Sample size $(E / C)$ & Adverse events cases & Experimental group (n) & Control group (n) \\
\hline Duan CR 2017 [13] & $36 / 36$ & $\mathrm{E}: 0 / 36 ; \mathrm{C}: 0 / 36$ & Reported no occurrence & Reported no occurrence \\
\hline Han DX 2014 [14] & $38 / 35$ & $\mathrm{E}: 2 / 38 ; \mathrm{C}: 7 / 35$ & $\begin{array}{l}\text { Hypertension; secondary infection } \\
\text { (Total 2) }\end{array}$ & $\begin{array}{l}\text { Hypertension; secondary infection; } \\
\text { mental symptoms (Total 7) }\end{array}$ \\
\hline Lu W 2015 [15] & $20 / 20$ & $\mathrm{E}: 6 / 20 ; C: 7 / 20$ & $\begin{array}{l}\text { Adverse reaction of glucocorticoid (2) } \\
\text { gastrointestinal discomfort (3) } \\
\text { respiratory tract infection (1) }\end{array}$ & $\begin{array}{l}\text { Adverse reaction of glucocorticoid (3) } \\
\text { gastrointestinal discomfort (3) } \\
\text { respiratory tract infection (1) }\end{array}$ \\
\hline Peng YH 2014 [16] & $28 / 26$ & E:NR; C:NR & NR & NR \\
\hline Shi Z 2019 [17] & $15 / 15$ & $\mathrm{E}: 1 / 15 ; C: 6 / 15$ & Diarrhea (1) & $\begin{array}{l}\text { Itching(1), diarrhea(2), rash(1), nausea(1), } \\
\text { dizziness (1) }\end{array}$ \\
\hline Yuan TT 2020 [18] & $47 / 47$ & E:NR; C:NR & NR & NR \\
\hline Zhou DJ a 2013 [19] & $10 / 10$ & $\mathrm{E}: \mathrm{NR} ; \mathrm{C}: \mathrm{NR}$ & NR & $N R$ \\
\hline Zhou DJ b 2013 [19] & $5 / 5$ & E:NR; C:NR & NR & NR \\
\hline
\end{tabular}

Abbreviations: $R C T$ s Randomized controlled trials, $H Q H$ Huaiqihuang, Experimental group, C Control group, NR Not reported

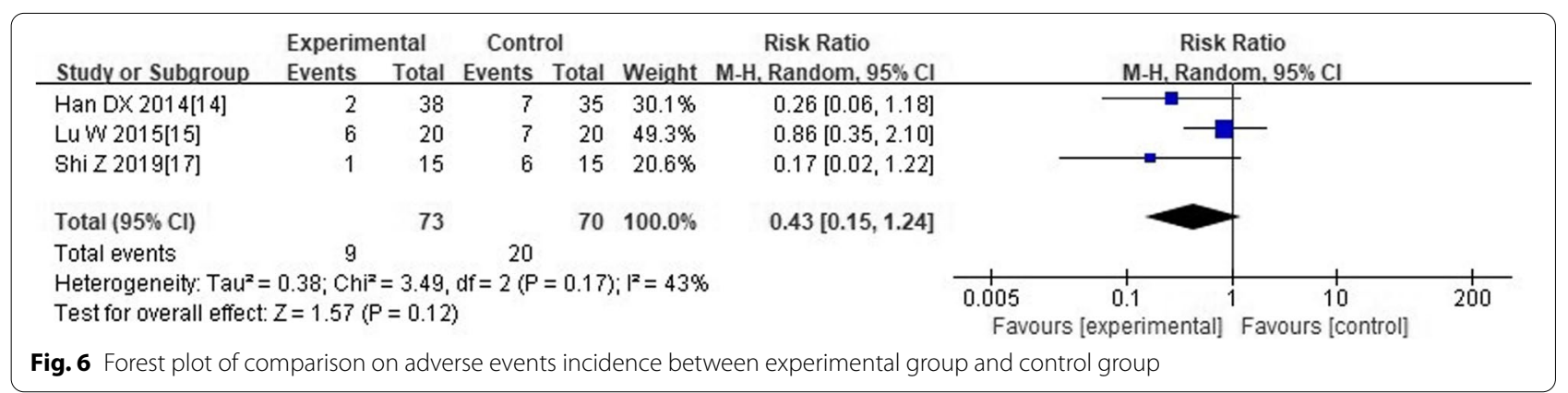

tends to exaggerate the effectiveness, the evidence may be not sufficient for clinical decision. We need to ensure enough power for the sample size estimation to enhance the precision and robustness of the result in future. As new studies may continue to be published, we should continue to update the systematic review on this topic, focusing on the above key clinical issues.

\section{Additional analysis}

We explored the source of heterogeneity for subgroup whose heterogeneity was significant. Forest plot of subgroup between "GC plus IS plus $\mathrm{HQH}$ " group and "GC plus IS" group on "clinical cure rate" manifested substantial heterogeneity of each trial (Fig. 3, $\mathrm{I}^{2}=67 \%$ ). After removing the research of Han DX [14], heterogeneity among the trials reduced ( $\mathrm{I}^{2}$ changed from 67 to $51 \%$ ). As well as another forest plot of subgroup between "GC plus IS plus HQH" group and "GC plus IS" group on "total effective rate" showed substantial heterogeneity of trials (Fig. $4, \mathrm{I}^{2}=73 \%$ ). Heterogeneity significantly declined $\left(\mathrm{I}^{2}\right.$ changed from 73 to $21 \%$ ) after excluding the trial of Han DX [14]. Considering that method of random sequence generation was unclear in Han DX's trial [14], and two immunosuppressive agents were used in this trial, the factors of significant heterogeneity may be related to the above situation.

Furthermore, we finished meaningful sensitivity analyses. After eliminating the research of Han DX [14], sensitivity analyses did not change the direction of results, including outcomes of "clinical cure rate" (RR 1.55; 1.17 to 2.05 ) and "total effective rate" (RR $1.27 ; 1.13$ to 1.42 ). The above suggested that results of the research could be consistent and robust.

Besides, since there was not enough (fewer than 10 articles) included trials, no publication bias test was performed.

\section{Discussion}

Principal findings and comparison with prior reviews

Although the literature has been comprehensively searched, we still have not found a systematic review on HQH's intervention in HSPN. Thus, we evaluated the effectiveness and safety of HQH for HSPN in children, so as to provide evidence for clinical use. A total of seven trials were included in this study. In addition to pooling the overall results, we also did subgroups analyses according to different conventional medicine interventions in order to minimize clinical 


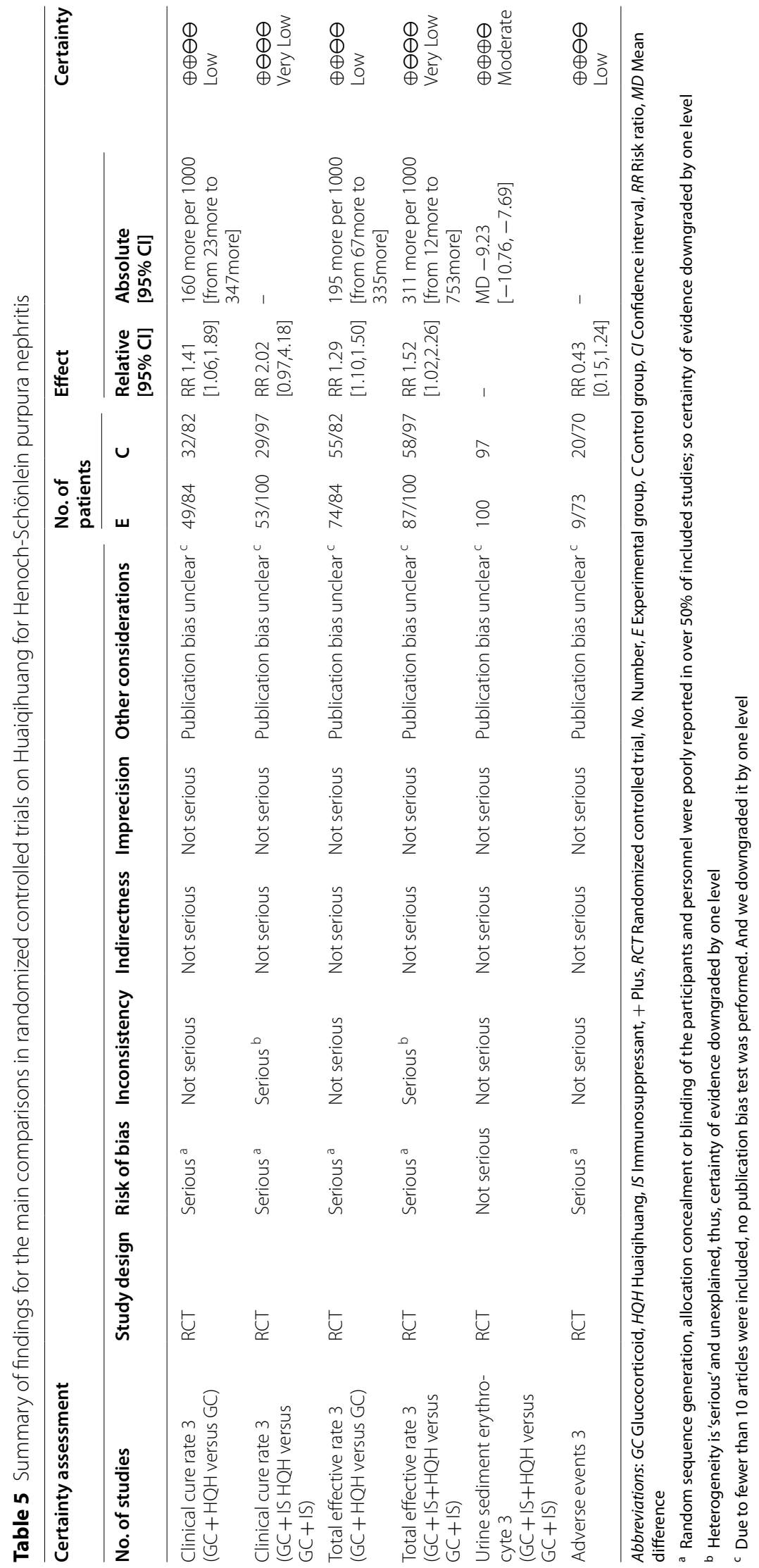


heterogeneity. Whether it was experimental group or control group, basic treatments were necessary. The application of GC or IS was based on clinical manifestation and pathological classification of the children. IS mainly included cyclophosphamide and mycophenolate mofetil. The results of our study demonstrated that compared with conventional medicine alone, the combined treatment of $\mathrm{HQH}$ and conventional medicine presented a superior effect in children with HSPN, including improving the clinical cure rate and total effective rate; reducing urinary protein excretion, urine sediment erythrocyte count and urine $\beta 2-\mathrm{MG}$ level. With regard to subgroup analysis of "clinical cure rate", despite the difference in cure rate between the two groups ("GC plus IS plus HQH" versus "GC plus IS") was not statistically significant, it was still necessary to be cautious when drawing conclusions. Perhaps because of small sample size, the test power was not enough to find the difference between the two groups. In general, additional administration of $\mathrm{HQH}$ appeared to strengthen the protection of glomerulus and tubules in children with HSPN.

Moreover, many researches have confirmed that immune abnormalities and inflammatory injury played a key role in pathogenesis of HSPN recently [34, 35]. $\mathrm{CD}^{+} \mathrm{T}$ cells are important cells involved in the immune response. Tregs and Th17 cells, which belong to $\mathrm{CD}^{+} \mathrm{T}$ cell subsets, maintain the body's immune balance. Tregs are responsible for the maintenance of self-tolerance, thus inhibiting autoimmunity, whereas pro-inflammatory Th17-cells contribute to the induction and propagation of inflammation [36]. In children with HSPN, an imbalance of Th17/Treg cell axis has been observed, which is often manifested as a decrease in Treg level and an increase in Th17 [37]. IL-17 is an important cytokine of Th17, which exerts immuno-suppressive effects [38]. IL-6 is an essential cytokine for the differentiation process of Th17 cells [39], and IL-4 is also the main pro-inflammatory factor [18]. Relevant studies have shown that the above-mentioned inflammatory factors were significantly elevated in children with HSPN. The pooling results of our study indicated that compared with conventional medicine alone, additional administration of $\mathrm{HQH}$ had better effect on elevating Treg (\%), lowering Th17 (\%) as well as lowering concentration of IL-6, IL-4 and IL-17. However, subgroups analyses results showed that compared with the "GC" group, co-intervention of HQH and GC did not have an advantage in increasing Treg and decreasing Th17 and IL-17. Maybe GC had powerful function of inhibiting the inflammatory factor IL-17, while the effect of $\mathrm{HQH}$ was masked. But considering extremely sparse sample size, the results need to be further confirmed in the future. Generally speaking, $\mathrm{HQH}$ seemed to regulate immune function and reduce inflammation damage in children with HSPN.

In terms of safety, the adverse events reported might be related to the side effects of medications under investigation. Adverse events observed mainly included hyper-tension, secondary infection, mental symptoms, gastrointestinal discomfort and so forth. In most cases, the reported abnormal symptoms were mild. There were no reports of serious adverse events in all the included studies. Combined with pooled results of meta-analyses, additional administration of $\mathrm{HQH}$ did not seem to increase the incidence of adverse events.

\section{Limitations}

We conducted a systematic searching and appraised the original studies critically. In spite of there were lots of trials on HQH treating HSP or HSPN, only seven trials met our inclusion criteria. Due to generally poor methodological quality of included trials and some outcome measures were mentioned in only one article, there was no richer evidence to support the results. In addition, there were certain differences in the types of immunosuppressants, the dosage and frequency of $\mathrm{HQH}$ administration, as well as the duration of treatment in included studies. Meanwhile, the literature generally did not report follow-up and relapse, and it was impossible to evaluate the long-term effectiveness, which may affect the reliability of the results. Apart from this, all the trials were carried out in China, so the limitation on the race of patients need to be considered. At last, there was not enough included trials, no publication bias test was performed. All the above limited the overall confidence of evidence, and our result may need to be explained with caution.

\section{Implications for further research}

As for GRADE assessment results, the overall certainty of evidence for main findings ranged from "very low" to "moderate". Consequently, highly quality RCTs with better design are necessary in the future to improve the level of evidence. Furthermore, considering the above limitations, it is also important to evaluate the long-term effects and safety of interventions in future studies. So adequate follow-up time is required to observe relapse and adverse events.

\section{Conclusions}

Chinese patent herbal medicine $\mathrm{HQH}$ combined with conventional medicine presented the beneficial effect for children with HSPN. And additional administration of $\mathrm{HQH}$ did not seem to increase the incidence of adverse events. However, considering the limited 
included studies and poor methodological quality, we should be cautious when using evidence in clinical practice. In the future, long term follow-up, high quality and multicenter RCTs are required to make the results more convincing.

\begin{abstract}
Abbreviations
HSP: Henoch-Schönlein purpura; HSPN: Henoch-Schönlein purpura nephritis; ACEls: Angiotensin-converting enzyme inhibitors; ARBs: Angiotensin receptor blockers; GC: Glucocorticoid; IS: Immunosuppressant; $\mathrm{HQH}$ : Huaiqihuang; RCTs: Randomized controlled trials; ISKDC: International Study of Kidney Disease in Children; BT: Basic treatment; $\beta 2-M G$ : $\beta 2$ micro-globulin; CNKI: China National Knowledge Infrastructure; VIP: Chinese Science and Technology Journal Database; IgAVN: Immunoglobulin A vasculitis with nephritis; GRADE: Grading of Recommendations Assessment, Development, and Evaluation; MD: Mean difference; $\mathrm{Cl}$ : Confidence interval; SMD: Standardized mean difference; RR: Risk ratio; ${ }^{2}:$ I-square; Pcs/HP: Pieces/ High-power field; Treg: Regulatory T cell; Th17: T helper cell 17; IL: Interleukin; E: Experimental group; C: Control group; NR: Not reported; +: Plus; Bid: Two times a day.
\end{abstract}

\section{Acknowledgments}

Centre for Evidence-Based Chinese Medicine of Beijing University of Chinese Medicine hosted the study and provided learning quidance to domestic visiting scholars. The author also thanks National Administration of Traditional Chinese Medicine for the base construction project.

\section{Authors' contributions}

Research idea and study design: XX and JPL. Protocol and Registration: XX and XHL. Search literature and data extraction: XX and QL. Methodology and software: $\mathrm{CLL}, \mathrm{XYJ}$, and JPL. Manuscript writing: $X X, X H L$ and $X Y$ J. Manuscript revision: XQW, CLL and JPL. Supervision: JPL. All authors have read and approved the final manuscript.

\section{Funding}

This work was supported by the key project of the National Natural Science Foundation of China (No.81830115). The funder had no role in study design, data collection and analysis, interpretation of result, or writing the manuscript.

\section{Availability of data and materials}

All data in our current study came from publicly available databases, including eight Chinese and English databases. And details related to this article are available from the corresponding author upon reasonable request.

\section{Declarations}

\section{Ethics approval and consent to participate}

All data in our study came from publicly available data. Our study didn't recruit human subjects. Therefore, ethical approval was not required. Not applicable.

\section{Consent for publication}

Not applicable.

\section{Competing interests}

All authors declare that they have no competing interests.

\begin{abstract}
Author details
'Hubei University of Traditional Chinese Medicine, The first clinical college and affiliated hospital, Wuhan 430061, Hubei, China. ${ }^{2}$ Beijing University of Chinese Medicine, Centre for Evidence-Based Chinese Medicine, Beijing 100029, China.
\end{abstract}

Received: 15 January 2021 Accepted: 20 September 2021

Published online: 08 November 2021

\section{References}

1. Zaffanello M, Brugnara M, Franchini M. Therapy for children with henoch-schonlein purpura nephritis: a systematic review. The Sci World J. 2007;7:20-30.

2. Nikibakhsh AA, Mahmoodzadeh H, Karamyyar M, Hejazi S, Noroozi M, Macooie AA, et al. Treatment of complicated henoch-schönlein purpura with mycophenolate mofetil: a retrospective case series report. Int J Rheumatol. 2010;2010:254316.

3. Ding Y, Zhang X, Ren X, Zhai W, He L, Liu J, et al. Traditional Chinese medicine versus regular therapy in Henoch-Schönlein purpura nephritis in children: study protocol for a randomized controlled trial. Trials. 2019;20:538

4. Wang $P$, Tang L, Yao J, et al. The spectrum of biopsy-proven secondary glomerular diseases: a cross-sectional study in China. Clin Nephrol. 2017:88:270-6.

5. Shi CC, Peng M, Du YY, et al. The efficacy and safety of integrated Chinese plus Western medicine compared with Western medicine alone in the treatment for purpuric nephritis: a Meta-Aalysis. Science. 2020;8:19-35.

6. Zou XR, Xue X, Wang CJ, et al. A Chinese cohort study on Henoch-schonlein purpura nephritis: design and methods. Eur J Inflamm. 2018;16:1-6.

7. Cattran DC, Feehally J, Cook HT, et al. Kidney disease: improving global outcomes (KDIGO) glomerulonephritis work group. KDIGO clinical practice guideline for glomerulonephritis. Kidney Int Suppl. 2012;2:139-274.

8. Zhang $X$, Cheng $Y$, Zhou Q, Huang H, Dong $Y$, Yang $Y$, et al. The effect of Chinese traditional medicine Huaiqihuang $(\mathrm{HQH})$ on the protection of nephropathy. Oxidative Med Cell Longev. 2020;16:2153912.

9. Li LT, Shi MY, Wei SY, et al. Huai qi Huang ameliorates proteinuria and hematuria in mild IgA nephropathy patients: a prospective randomized controlled study. J Formos Med Assoc. 2013;112:766-72.

10. Liu H, Sun W, Tu Y. Mechanism of Huaiqihuang granules delaying chronic kidney disease. Chin Nephrol J Integr Tradit West Med. 2014:15:926-8.

11. Geng HY, Cao L, Wang W, et al. A prospective, randomized, controlled clinical study of Huaiqihuang granules in treatment of childhood primary nephrotic syndrome. Chin J Contemp Pediatr. 2015;17:31-4.

12. DuY, Hu ZJ, Hou L, et al. Clinical observation of Huaiqihuang granules combined with glucocorticoid in treating primary IgA nephropathy in children. Liaoning J Tradit Chin Med. 2018;45:328-31.

13. Duan $C R$, Li ZH, Zhang Y, et al. Effects of Huaiqihuang granules on hemorheology and serum hs-CRP in children with HSPN. Guid J Tradit Chin Med Pharm. 2017:23:94-5+8.

14. Han DX. Clinical observation on treatment of 38 cases of pediatric HSPN with integrated traditional Chinese and conventional medicine. Chin J Ethno Med Pharm. 2014;23:42+4.

15. Lu W. Effect of Huaiqihuang granules on serum cytokines in children with HSPN. Chin J Pharm Ind. 2015;24:47-9.

16. Peng YH, Deng LL. Effect of Huaiqihuang granules in treating HSPN and its influence on serum cytokines. J Community Med. 2014;12:10-2.

17. Shi $Z$, Weng $Z Y$, Yu L. Clinical study of Huaiqihuang granules combined with glucocorticoid and mycophenolate mofetil in the treatment of children with purpuric nephritis. Int Med Health Guid News. 2019;15:2483-7.

18. Yuan TT, Zhang RF. The effect of Huaiqihuang granule on children with HSPN and its influence on renal function and immune inflammatory factors. Mod J Integr Tradit West Med. 2020;29:545-8.

19. Zhou DJ, Wu XC, Wang FJ, et al. The regulating effect of Huaiqihuang on Th17/Treg cell immune imbalance in children with purpuric nephritis. Chin J Pract Pediatr. 2013;5:352-6.

20. Wang Z, Chen Y, Luo J, et al. Observation of curative effect and mechanism of Huaiqihuang intervention in mouse IgA nephropathy model. Chin Nephrol J Integr Tradit West Med. 2011;12:974-8+1038.

21. Li T, Mao J, Huang L, Fu H, Chen S, Liu A, et al. Huaiqihuang may protect from proteinuria by resisting MPC5 podocyte damage via targeting p-ERK/CHOP pathway. Bosnian J Basic. 2016;16:193.

22. Sun W, Zhu Z, Yu J, et al. Effect of Huaiqihuang granules on the expression of nephrin and podocin in the renal tissue of rats with adriamycin nephropathy. J Integr Med. 2011;9:546-52.

23. Lu HY, Zhang QL, Jiang XY, et al. The effect of Huaiqihuang on proteinuria, nephrin and podocin in kidney tissue of rats with IgA nephropathy. Chin J Obstet Gynecol Pediatr. 2009;5:458-65.

24. Bai J, Geng W, Mei Y, et al. Effect of Huaier on the proliferation of mesangial cells in anti-Thy-1 nephritis. Cell Physiol Biochem. 2017;42:2441-52. 
25. Pu J, Zhang Y, Zhou J. Effect of Huai Qi Huang on epithelial-mesenchymal transition of renal tubular epithelial cells through miR-200a. Evid Based Compl Alt. 2016;2016:8612190.

26. Moher D, Liberati A, Tetzlaff J, Altman DG, Prisma Group. Preferred reporting items for systematic reviews and meta-analyses: the PRISMA statement. PLoS Med. 2009;6:e1000097.

27. Nephrology Group, Pediatrics Society, Chinese Medical Association. Evidence-based guidelines for the diagnosis and treatment of common kidney diseases in children (II): evidence-based guidelines for the diagnosis and treatment of Henoch-Schonlein purpura nephritis. Chin J Pediatr. 2009:47:911-3.

28. Ye RG, Chen YS, Fang JA. Summary of discussions on diagnosis, treatment and curative effect standards of nephropathy. Chin J IntegrTradit Western Nephrol. 2003:4:355-7.

29. Cumpston M, Li T, Page MJ, Chandler J, Welch VA, Higgins JP, et al. Updated guidance for trusted systematic reviews: a new edition of the Cochrane handbook for systematic reviews of interventions. Cochrane Database Syst Rev. 2019:10:ED000142.

30. Chen W, Fang SN, Liu JP, et al. The development and current situation of international evidence-based medicine evidence classification system. Chin Nephrol J Integr Tradit West Med. 2017;37:1413-9.

31. Julian Higgins, James Thomas. Cochrane handbook for systematic reviews of interventions version 6.0.2019. https://training.cochrane.org/ handbook. Accessed 21 Dec. 2020

32. Wang N, Liu L, Li CZ, et al. Study on the effect of Huaiqihuang granule on T lymphocyte subsets and urine kidney damage molecule-1 in HSPN. Chin J Pract Pediatr. 2013;28:943-5.
33. Ma XL. Clinical observation of Huaiqihuang granule in children with Henoch-schonlein purpura nephritis. Hebei Univ Med Sci. 2019;3:1-35.

34. Yang B, Tan X, Xiong X, et al. Effect of CD40/CD40L signaling on IL-10-producing regulatory $B$ cells in Chinese children with Henoch-Schönlein purpura nephritis. Immunol Res. 2017:65:592-604.

35. Shao $X$, Jiang $C$, Li Y, et al. Function of $C D 4(+) C D 25(+)$ regulatory $T$ cells in Henoch-Schonlein purpura nephritis in children. Chin J Pediatr. 2014;52:516-20.

36. Fasching P, Stradner M, Graninger W, Dejaco C, Fessler J. Therapeutic potential of targeting the Th17/Treg Axis in autoimmune disorders. Molecules. 2017;22:134.

37. Chen O, Zhu XB, Ren H, et al. The imbalance of Th17/Treg in Chinese children with Henoch-Schonlein purpura. Int Immunopharmacol. 2013:16:67-71.

38. Xue X, Liu Q, Wang XQ, et al. Correlation between expression of Treg/ Th17 cell Axis in peripheral blood and TCM syndrome type in adults with HSPN patients. Mod Tradit Chin Mater Med—World Sci Technol. 2020;22:2907-13.

39. Shao XS, Jiang $C$, Li Y Y et al. Study on the function of CD4+CD25+ regulatory T cells in children with purpuric nephritis. Chin J Pediatr. 2014;52:516-20.

\section{Publisher's Note}

Springer Nature remains neutral with regard to jurisdictional claims in published maps and institutional affiliations.
Ready to submit your research? Choose BMC and benefit from:

- fast, convenient online submission

- thorough peer review by experienced researchers in your field

- rapid publication on acceptance

- support for research data, including large and complex data types

- gold Open Access which fosters wider collaboration and increased citations

- maximum visibility for your research: over $100 \mathrm{M}$ website views per year

At BMC, research is always in progress.

Learn more biomedcentral.com/submissions 